\title{
A Hybrid Mobile Robot Path Planning Scheme Based on Modified Gray Wolf Optimization and Situation Assessment
}

\author{
Yilun Liu $(\mathbb{D}$ and Xiaoming Li $(D)$ \\ School of Electrical and Information, Northeast Agricultural University, Harbin 150030, China \\ Correspondence should be addressed to Xiaoming Li; lixiaoming@neau.edu.cn
}

Received 7 December 2021; Revised 10 January 2022; Accepted 17 January 2022; Published 16 February 2022

Academic Editor: Shan Zhong

Copyright (๑) 2022 Yilun Liu and Xiaoming Li. This is an open access article distributed under the Creative Commons Attribution License, which permits unrestricted use, distribution, and reproduction in any medium, provided the original work is properly cited.

\begin{abstract}
To better solve the problems associated with optimal pathfinding and dynamic obstacle avoidance in the path planning of mobile robots, a hybrid path planning scheme combining modified gray wolf optimization (MGWO) and situation assessment mechanism is proposed. Firstly, a MGWO algorithm is proposed to plan a global path. Secondly, different situational factors for robots in different regions are extracted from the fusion results of 2D laser measurements and image data, and a Bayesian network model of robot action selection is established. Then, the situational factors of the robot are used as evidence for reasoning. Based on the posterior probability value in the inference result, the grid to be moved is selected and the traveling direction of the robot is adjusted in order to take advantage of both global path planning and local dynamic obstacle avoidance. The simulation results show that the proposed MGWO has better optimization performance. When combined with a situation assessment mechanism, it realizes dynamic obstacle avoidance while keeping the path length as short as possible.
\end{abstract}

\section{Introduction}

With technological advancement and social development, the level of intelligence and automation of mobile robots has gradually improved, and it has gradually penetrated into people's daily life [1]. In the field of mobile robots, path planning is one of the most important requirements, which is the key technology to realize autonomous robot navigation [2].

The mobile robot needs to plan a short, energy-efficient, and safe path from the initial position to the target position, and it must be able to avoid static and dynamic obstacles along the way. At the same time, mobile robots should have certain computing capabilities of calculating the shortest and safest path in real time to reduce time and energy consumption [3].

This article proposes a mobile robot path planning scheme based on the MGWO and situation assessment mechanism. Firstly, a global path is planned with the proposed MGWO. Secondly, a situation assessment mechanism is used to fuse the data from multiple sensors equipped on the robots, obtaining situational factors from multisensor information and inferring the impacts of the detected obstacles on the robot movement so as to determine the next move. Moreover, the corresponding action is carried out to find the shortest path or the second shortest path from the starting position to the destination without any collision with the obstacles.

The contributions of this article are listed as follows:

(1) To solve the problem of global path planning of the mobile robot, a modified gray wolf optimization algorithm (MGWO) is proposed in which the population diversity is enhanced by logistic chaotic mapping. An adaptive adjustment strategy of control parameters is utilized to achieve a balance between search and development capabilities. With the static weighting average strategy, the population position is updated to speed up the convergence speed, thereby boosting the performance in finding an optimal global path.

(2) From the perspective of cognition, the mechanism of a situation assessment is introduced to the 
application of mobile robots, which provides a new solution for the local path planning of mobile robots.

(3) A hybrid planning scheme based on the divide-and conquer concept is proposed. The initial path based on global environment information is obtained with the MGWO algorithm. When an obstacle is detected, the situation assessment mechanism is used to avoid the obstacle through local planning and then back to the global path. Repeat the process until the destination is reached.

The rest of this article is organized as follows. Section 2 introduces the related path planning methods. The details of the proposed MGWO are explained in Section 3. Section 4 describes the proposed situation assessment mechanism. In Section 5, a comprehensive comparative analysis is conducted based on the results obtained from a number of experiments. Finally, Section 6 concludes the full article.

\section{Related Works}

According to the amount of situational information acquired, robot path planning methods can be divided into two categories: global path planning and local path planning, where local path planning is also called dynamic path planning [4].

At present, global path planning algorithms can be generally classified as conventional algorithms and intelligent algorithms. The former includes $A^{*}$ algorithm [5] and RRT algorithm [6], and the latter includes ant colony algorithm [7] and particle swarm algorithm [8]. Traditional algorithms mostly use the length of the path as the only indicator, while intelligent search algorithms use randomly generated initial solutions or sampling points to approximate the optimal solution through multiple iterations. The biggest characteristic of an intelligent algorithm is its randomness, so its solution is not unique. A lot of heuristic intelligent search methods have been proposed with respect to optimization algorithms, considering that there are a large number of NP-hard problems in path planning, and each problem has its own optimal solution, which may not be solved. Therefore, the heuristic intelligent search algorithm approximates the optimal path through iterative improvements of randomly generated initial feasible solutions [9].

Since its proposal, the gray wolf optimization (GWO) [10] algorithm has been widely studied because of its advantages of good searching performance, simple structure, and easy implementation. For example, Toufan and Niknafs [11] exploited the cosine function-based searching factors and introduced a dynamic weighting strategy to balance global and local exploring abilities, which improves the solution accuracy of GWO. Olivera et al. [12] used an exponential function to attenuate the searching factor $\alpha$ so that the convergence factor is changed nonlinearly and dynamically with the increase of iteration times and tried to find a better trade-off between the search and development stages to ensure that the optimal solution is approached. Albina and Lee [13] proposed balancing the searching ability with stochastic convergence factors and performing individual update through differential mutation to improve the convergence and accuracy of the original GWO.

The proposed MGWO further improves the performance of the original GWO and speeds up the convergence. However, as a global path planning method, the MGWO does not have the ability to avoid obstacles in real time. Aiming at this problem, we propose a dynamic path planning of mobile robots based on the fusion of MGWO and a situation assessment mechanism.

Local path planning is a kind of dynamic planning. The robot perceives its surroundings with sensor information and plans a collision-free path from the current node to a target subnode online. In a rapidly changing environment, it is necessary to continuously collect sensor information and replan the path to guide the robot's movement in real time. The planning range is usually limited to the detection range of the sensors. Commonly used local planning algorithms include artificial potential field [14], dynamic window [15], $D^{*}$ algorithm [16], and fuzzy logic approaches [17].

Situation assessment is the high-level information fusion in a multisensor information system. It is the extraction, combination, and comprehensive processing of the highlevel relationships of the information flows of different types and multiple levels obtained from the system, thereby acquiring the important cognitive information from sensor information to infer the target intentions and future trends. Situation assessment is a cognitive reasoning method based on human thinking.

Huang et al. [18] proposed a cyber situational awareness (CSA) decision-making system based on fuzzy sets, in which a team CSA with the ability to process unknown information is established in a distributed way. Arora et al. [19] proposed to code certain knowledge with a Bayesian network and perform network reasoning by Monte Carlo tree search technique to plan actions based on information perception. The experiment results prove the method has practical value. Zhou et al. [20] proposed an uncertain information fusion method for aerial situation awareness in the DST framework. Firstly, the perceived uncertain information is preprocessed with belief entropy, and the evaluated information is combined according to Dempster combination rules. This kind of technique can enhance and expand the limited cognitive ability.

\section{Global Path Planning with MGWO}

3.1. Problem Formulation. Given the regional environment map. $\mathbf{T}=\left\{\mathbf{T}_{1}, \mathbf{T}_{2}, \ldots, \mathbf{T}_{n}\right\}$ is the collection of target points. $\mathrm{O}$ is the starting point of the robot. The robot is required to traverse $n$ checkpoints and return to the starting point. The traversal path is $\mathbf{R}=\left\{\mathbf{O}, \mathbf{T}_{1}, \mathbf{T}_{2}, \ldots, \mathbf{T}_{n}, \mathbf{O}\right\}$. Then, the path length $L$ can be calculated as follows:

$$
L=\left\|\mathbf{O} \mathbf{T}_{1}\right\|+\sum_{j=1}^{n-1}\left\|\mathbf{T}_{j} \mathbf{T}_{j+1}\right\|+\left\|\mathbf{T}_{n} \mathbf{O}\right\|,
$$

where $\left\|\mathbf{O T} \mathbf{T}_{1}\right\|$ denotes the distance traveled by the robot from the starting point to the first checkpoint. $\left\|\mathbf{T}_{j} \mathbf{T}_{j+1}\right\|$ is the distance from checkpoint $j$ to checkpoint $j+1$. $\left\|\mathbf{T}_{n} \mathbf{O}\right\|$ is the 
distance from checkpoint $n$ to the starting point. It is required to find the order of the traversal checkpoints $\left\{\mathbf{T}_{1}, \mathbf{T}_{2}, \ldots, \mathbf{T}_{n}\right\}$ so that the shortest path is found with length $\min L$.

3.2. Classic Gray Wolf Optimization. The gray wolf optimization is an algorithm that simulates the predation behaviors of predator wolves at the top of the food chain. Most gray wolves like to live in groups and have a very strict social hierarchy, as shown in the pyramid structure of Figure 1.

In the mathematical model of GWO, each wolf represents a candidate solution in the group, where $\alpha$ is the optimal solution and $\beta$ and $\delta$ are the second and third best solution, respectively; the rest of candidate solutions are denoted by $\omega$. In GWO, the search (optimization) is guided by $\alpha, \beta$, and $\delta$ and followed by $\omega$.

Let the population size of the gray wolves be $N$, and the search space is a Dim-dimensional space, $\mathbf{X}_{i}^{\text {Dim }}$ is the position vector of the $i$ th wolf in the Dim-th dimension; then, the mathematical model of the gray wolves hunting prey is expressed as follows:

$$
\begin{aligned}
\mathbf{D} & =\left|\mathbf{C X}_{p}(t)-\mathbf{X}(t)\right|, \\
\mathbf{X}(t+1) & =\mathbf{X}_{p}(t)-\mathbf{A D},
\end{aligned}
$$

where $t$ is the current iteration number. $\mathbf{A}$ and $\mathbf{C}$ are coefficient vectors, $\mathbf{A}=2 \mathbf{a r}_{1}-\mathbf{a}, \mathbf{C}=2 \mathbf{r}_{2}$, where $\mathbf{r}_{1}$ and $\mathbf{r}_{2}$ are random numbers in the range $[0,1] . a$ is the convergence factor, and its value linearly decreases from 2 to 0 as the number of iterations increases. $\mathbf{X}_{p}$ is the position vector of the prey. $\mathbf{D}$ is the positional relationship vector between the gray wolf individual and the prey. $\mathbf{X}$ is the position vector of the gray wolf.

The mathematical model of the gray wolves tracking prey locations can be expressed as follows:

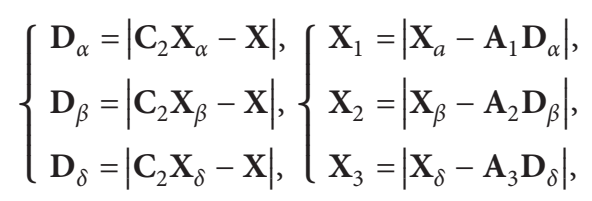

where $\mathbf{C}_{1}, \mathbf{C}_{2}$, and $\mathbf{C}_{3}$ are random vectors. $\mathbf{D}_{\alpha}, \mathbf{D}_{\beta}$, and $\mathbf{D}_{\delta}$ denote the distance of wolves $\alpha, \beta$, and $\delta$ with other members in the wolf pack, respectively. $\mathbf{X}_{\alpha}, \mathbf{X}_{\beta}$, and $\mathbf{X}_{\delta}$ denote the positions of wolves $\alpha, \beta$, and $\delta$, respectively. $\mathbf{X}$ is the current position of the wolf pack. The position of wolf $\omega$ in the wolf pack is jointly determined by wolves $\alpha, \beta$, and $\delta$.

\subsection{Modified Gray Wolf Optimization Algorithm}

3.3.1. Population Initialization Based on Logistic Mapping. Chaos has the characteristics of randomness, ergodicity, and regularity. By introducing chaotic sequences, the individuals in the initial population can utilize the information in the solution space as much as possible, thereby enhancing global searching capability. In the population initialization stage, chaotic sequences are generated using logistic mapping [21], which is simple and chaotic and has good traverse

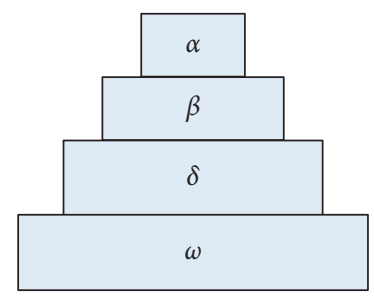

FIGURE 1: Social hierarchy of gray wolves.

uniformity to generate chaotic sequences to initialize the population position of the wolf pack. The logistic mapping can be expressed as follows:

$$
x_{n+1}=a x_{n}\left(1-x_{n}\right)
$$

where $x_{n} \in[0,1]$ is the chaotic variable, $a=4$. Let $x_{0}=0.4288$. Figure 2 shows the bubble map after the population initialization of 30 populations using logistic mapping.

\subsubsection{Adaptive Adjustment Strategy of the Control} Parameters. The global searching ability and local development ability of the GWO algorithm can be controlled by $a$. It decays from 2 to 0 linearly as the number of iterations increases, and the iterative convergence process is not linear. Based on this fact, we propose an adaptive adjustment strategy for nonlinear control parameters, which balances the search and development capabilities of the GWO algorithm by adjusting the value of $a$ adaptively. In early iterations, when the global search is performed, a larger value is assigned to $a$ to realize a fast nonlinear changing rate and powerful global search ability and avoid local optimum. In later iterations, a smaller value is assigned to $a$ to slow down the nonlinear changing rate in order to find an optimal solution within a certain region, thereby achieving strong development ability and boosting convergence speed.

The strategy can be expressed as follows:

$$
a(t)=a_{\text {initial }}-a_{\text {initial }}\left[\frac{1}{e-1}\left(e^{\left(t / t_{\max }\right)}-1\right)\right]^{n},
$$

where $t$ is the current iteration times. $T_{\max }$ is the maximum number of iterations. $n \in[1,2]$ is the linear modulation index. $a_{\text {initial }}$ is the initial value of the control parameter $a$, $a_{\text {initial }}=2$. Figure 3 shows the nonlinear decaying trend of $a$.

3.3.3. Static Weighting Average Strategy. The main idea of the static weighting average strategy is to weigh three leader wolves in accordance with the hierarchical structure of the pyramid. Using static weighting average strategy, wolves $\alpha, \beta$, and $\delta$ are given the weights of $0.5,0.3$, and 0.2 , respectively:

$$
\mathbf{X}(t+1)=\frac{5 \mathbf{X}_{1}+3 \mathbf{X}_{2}+2 \mathbf{X}_{3}}{10}
$$

\section{Situation Assessment}

In the proposed situation assessment mechanism, the situational factors of the environment where the robot is 


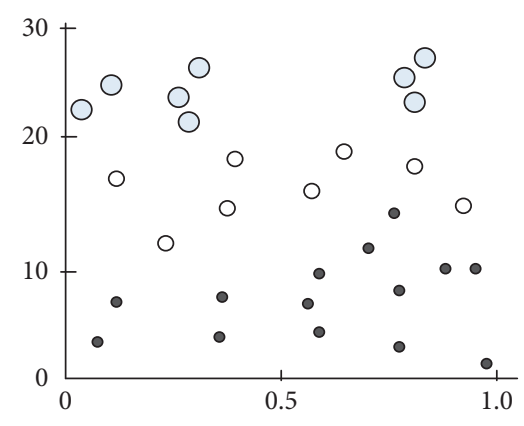

FIgURE 2: Population initialization with logistic mapping.

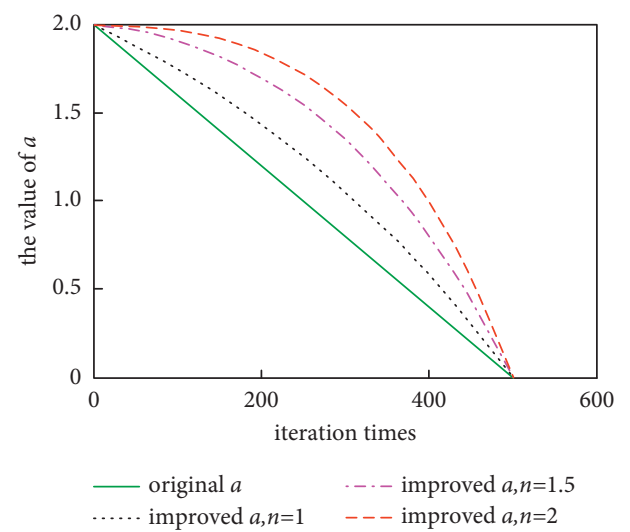

Figure 3: Comparison of the control parameter $a$.

located are extracted based on the $2 \mathrm{D}$ laser ranging data, sonar sensor measurements, and 2D image data of the mobile robot in order to infer the next move of the robot, thereby realizing local path planning within the unknown environment.

4.1. Situational Factor Extraction. Through the fusion of $2 \mathrm{D}$ laser ranging data and monocular camera image data, the target information located at the forefront of the mobile robot, that is, within the range of $\left[1^{\circ}, 17^{\circ}\right]$, is obtained.

Figure 4 shows the coordinate system of the mobile robot, where the positive and negative directions of axis $X t$ represent the front and back directions of the robot movement, respectively. The positive and negative directions of axis $Y t$ represent the right and left directions of the robot movement, respectively.

Based on the distance $D$ between the target and the robot and the angle $A\left({ }^{\circ}\right)$ between the target and the positive direction of axis $Y t$ of the robot coordinate system, the environmental factors are extracted using 2D laser ranging data. Place the angle $A$ of the location information into a certain region in the map; if the distance $D$ satisfies $0 \leq D \leq 220$, then the target is regarded as a situational factor of the region.

4.2. Bayesian Network Model of Robot Action Selection. In this study, the local path planning of a mobile robot is classified into three types of relatively independent action

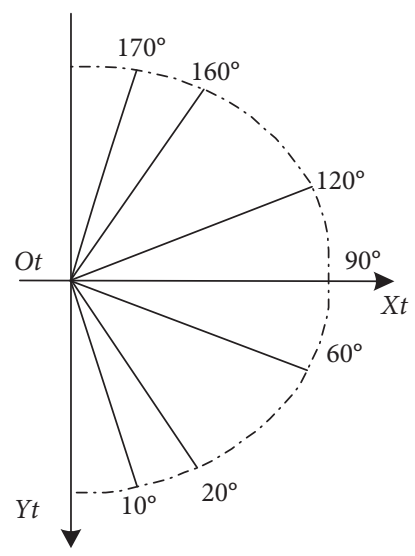

FIgURE 4: Situational factor extractions based on angle partition.

units (subactions): moving forward, obstacle avoidance, and escaping from a U-shaped trap.

Taking the robot coordinate system in Figure 4 as a reference, the discrimination rules of the three types of subactions are determined. For example, if there are obstacles in the right-ahead, right-front, and left-front directions of a mobile robot, then the robot will choose the subaction of obstacle avoidance.

The forebode node and the result node are extracted from the antecedent part and subsequent part of the discriminant rules, respectively, as listed in Table 1.

In this study, the obstacles in the right and left directions will be regarded as parallel to the moving direction of the robot. Therefore, the forebode node $T_{2}$ in Table 1 is determined based on the obstacles with respect to the robot position, that is the right or left obstacles. By using the diagnostic Bayesian network modeling, that is, take the forebode node as the father node of the result node and take the result node as the child node of the forebode node, the Bayesian network model of action selections is established as shown in Figure 5.

In Figure 5, the action selection node $C$ comprehensively considers the values of the forward node, avoidance node, and escaping node. Except for node $C$, all nodes in Figure 5 are of the discrete node type, which are binary discrete nodes, and their value states are yes $(Y)$ and no $(N)$. Node $C$ is a four-value discrete node, and its value states are $S_{0}, S_{1}, S_{2}$, and $S_{3}$. Among them, $S_{0}$ means choosing to move forward, $S_{1}$ means choosing to avoid obstacles, $S_{2}$ means choosing to escape from U-shaped traps, and $S_{3}$ means an error state. After determining the Bayesian network structure, the conditional probabilities of each node need to be given. The parameter is configured based on the discriminate rules of subactions. Take the avoidance node $\left(S_{2}\right)$ as an example; the configuration is listed in Table 2.

All forebode nodes in Figure 5 are the root nodes of the Bayesian network. In this study, the value of all forebode nodes is set to $(0.5,0.5)$.

4.3. Action Selection Inference. For the action selection inference of the mobile robot, based on the Bayesian network model shown in Figure 5, inputting the evidence and 
TABLE 1: Robot action selection based on Bayesian network model.

\begin{tabular}{|c|c|c|}
\hline Node description & Node type & Explanation \\
\hline Obstacle judging node $\left(T_{1}\right)$ & $\begin{array}{l}\text { Forebode } \\
\text { node }\end{array}$ & $\begin{array}{l}\text { Whether or not there is an obstacle in front of the robot within the range }\left[1^{\circ} \text {, }\right. \\
\left.\qquad 17^{\circ}\right]\end{array}$ \\
\hline $\begin{array}{l}\text { Obstacle node in parallel with the robot } \\
\left(T_{2}\right)\end{array}$ & $\begin{array}{l}\text { Forebode } \\
\text { node }\end{array}$ & $\begin{array}{l}\text { Whether or not the obstacle detected is parallel to the moving direction of the } \\
\text { robot }\end{array}$ \\
\hline Right-front obstacle $\left(T_{3}\right)$ & $\begin{array}{l}\text { Forebode } \\
\text { node }\end{array}$ & Whether or not there is an obstacle on the right front of the robot \\
\hline Left-front obstacle $\left(T_{4}\right)$ & $\begin{array}{l}\text { Forebode } \\
\text { node }\end{array}$ & Whether or not there is an obstacle on the left front of the robot \\
\hline Right-ahead obstacle $\left(T_{5}\right)$ & $\begin{array}{l}\text { Forebode } \\
\text { node }\end{array}$ & Whether or not there is an obstacle right ahead of the robot \\
\hline Right obstacle $\left(T_{6}\right)$ & $\begin{array}{l}\text { Forebode } \\
\text { node }\end{array}$ & Whether or not there is an obstacle on the right of the robot \\
\hline Left obstacle $\left(T_{7}\right)$ & $\begin{array}{l}\text { Forebode } \\
\text { node }\end{array}$ & Whether or not there is an obstacle on the left of the robot \\
\hline Forward node $\left(S_{1}\right)$ & Result node & If the robot chooses to move forward \\
\hline Avoidance node $\left(S_{2}\right)$ & Result node & If the robot chooses to avoid obstacle \\
\hline Escaping node $\left(S_{3}\right)$ & Result node & If the robot chooses to escape from a U-shaped trap \\
\hline Action selection node $\left(S_{4}\right)$ & Result node & The final action decision made by the robot \\
\hline
\end{tabular}

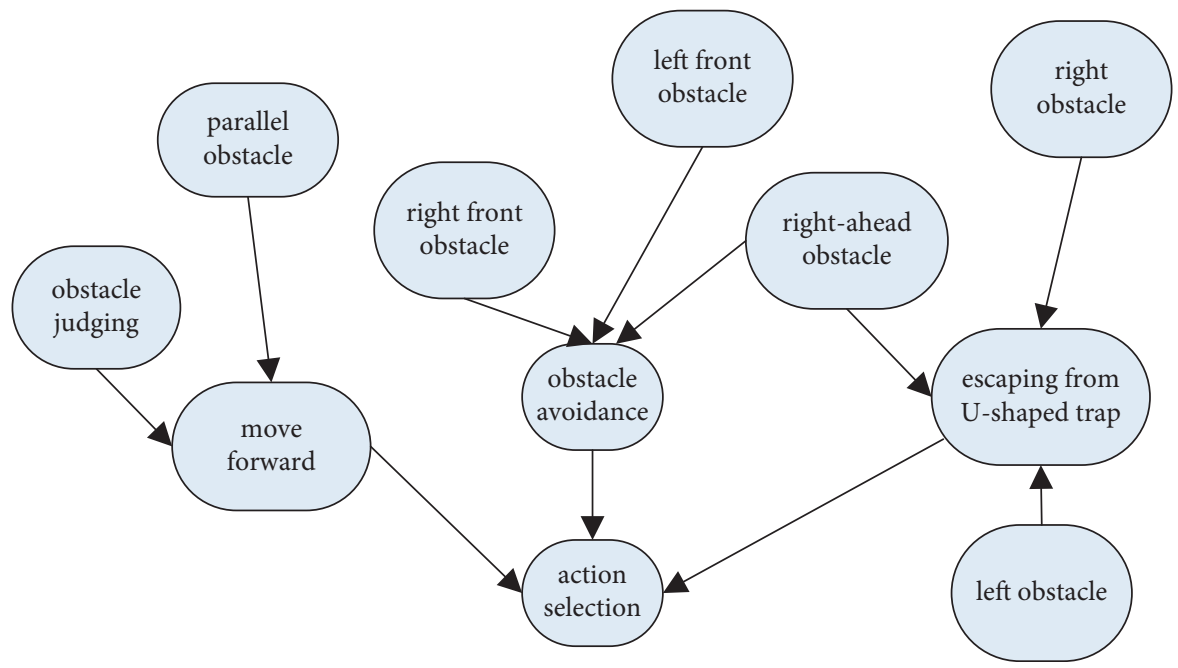

FIGURE 5: Bayesian network model.

TABle 2: Parameter configuration for $\mathrm{S}_{2}$ node.

\begin{tabular}{lcccc}
\hline$T_{3}$ & $T_{4}$ & $T_{5}$ & $\mathrm{Y}$ & $S_{2}$ \\
\hline $\mathrm{Y}$ & $\mathrm{Y}$ & $\mathrm{Y}$ & 1.0 & $\mathrm{~N}$ \\
$\mathrm{Y}$ & $\mathrm{Y}$ & $\mathrm{N}$ & 1.0 & 0.0 \\
$\mathrm{Y}$ & $\mathrm{N}$ & $\mathrm{Y}$ & 1.0 & 0.0 \\
$\mathrm{Y}$ & $\mathrm{N}$ & $\mathrm{N}$ & 1.0 & 0.0 \\
$\mathrm{~N}$ & $\mathrm{Y}$ & $\mathrm{Y}$ & 1.0 & 0.0 \\
$\mathrm{~N}$ & $\mathrm{Y}$ & $\mathrm{N}$ & 1.0 & 0.0 \\
$\mathrm{~N}$ & $\mathrm{~N}$ & $\mathrm{Y}$ & 1.0 & 0.0 \\
$\mathrm{~N}$ & $\mathrm{~N}$ & $\mathrm{~N}$ & 0.0 & 0.0 \\
\hline
\end{tabular}

calculating the posterior probabilities of the selection nodes by the reasoning algorithm of the Bayesian network, the result node with the biggest posterior probability will be chosen as the action decision of the robot.
In the Bayesian network of action selection, the forebode nodes are evidence nodes, and evidence values need to be assigned to the corresponding forebode nodes before performing the inference.

The evidence values of the forebode nodes are obtained from the extracted situational factors of the robot. For example, if the set of situational factors in the right-ahead direction is not empty, then the evidence value of the obstacle node $T_{5}$ in the right-ahead direction is set as $Y$; otherwise, it is set to $N$. Based on the extracted situational factors, input evidences for seven forebode nodes $\left(T_{1}, T_{2}, T_{3}\right.$, $T_{4}, T_{5}, T_{6}, T_{7}$ ), where the evidence values are denoted by $e 1$, $e 2, \ldots, e 7$, form an evidence set $E=\left\{e_{1}, e_{2}, \ldots, e_{7}\right\}$. Taking the evidence set $E$ as posterior conditions, the posterior probabilities of the action selection node $C$ can be calculated with Bayesian network reasoning algorithm; then, the posterior probabilities of node $C$ in 4 value states can be 
TABle 3: Action selection details.

\begin{tabular}{lcr}
\hline & Subaction & Deviation angle $\left(/{ }^{\circ} \mathrm{C}\right)$ \\
\hline 1 & Move forward & 0 \\
2 & Escaping from U-shaped obstacle & 30 to the right \\
& & 60 to the right \\
& & 30 to the left \\
3 & Obstacle avoidance & 30 to the left the left or right \\
& & 60 to the left or right \\
& & 0 \\
\hline
\end{tabular}

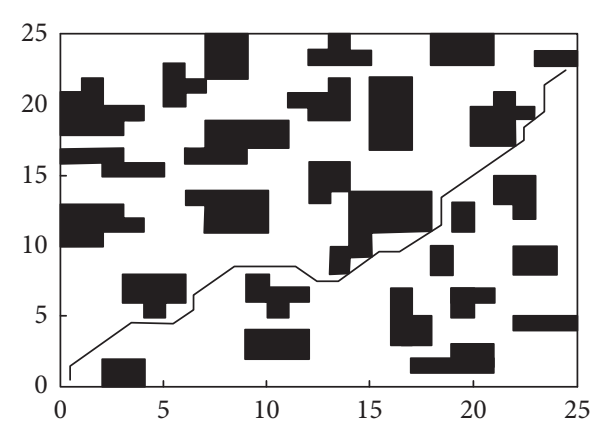

(a)

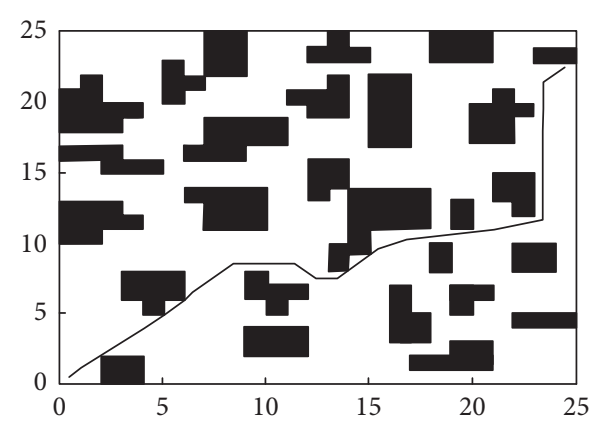

(b)

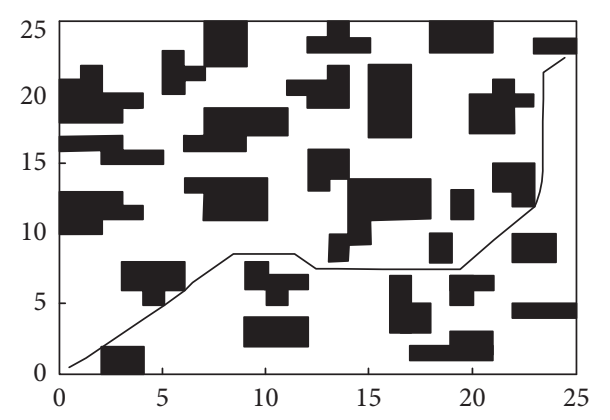

(c)

FIGURe 6: Path planning performance of different methods. (a) Path planned by the PSO algorithm. (b) Path planned by the GWO algorithm. (c) Path planned by the MGWO algorithm.

obtained as follows: $\quad P\left(C=S_{0} / E\right), \quad P\left(C=S_{1} / E\right)$, $P\left(C=S_{2} / E\right), P\left(C=S_{3} / E\right)$.

The Bayesian network reasoning algorithms include precise reasoning algorithm and approximate reasoning algorithm. Due to the low complexity of the Bayesian network model of action selection, this article adopts the precise reasoning algorithm of the Bayesian network, which is the variable elimination method.

Certain movement decisions will be taken in corresponding to the subaction chosen by the mobile robot for the next step. The decisions for the three types of subactions are listed in Table 3.

4.4. Hybrid Path Planning. The fundamental idea behind the hybrid path planning mechanism is based on the divideand-conquer concept. The mechanism is divided into two scenarios. Under the condition that the global situation information is known, a global path is planned with the global path planning algorithm. On this basis, the situation assessment method is used to plan a local path to avoid obstacles.

Firstly, an initial path based on global situation information is planned with the proposed MGWO algorithm. Local path planning is carried out under the guidance of the global path. When there are no obstacles that randomly appear in the surroundings, the situation assessment method plans a trajectory along the global path. If random obstacles are detected nearby that block the robot from continuing to move along the global path, the situation assessment method focuses on obstacle avoidance, planning a local path to avoid the obstacles. After that, the robot returns back to the global path until it reaches the destination.

\section{Experiment}

5.1. Global Path Planning Test. Firstly, the grid map is used to model the environment information, and the global path 
TABLE 4: Quantification results.

\begin{tabular}{lcccc}
\hline Methods & Longest path & Shortest path & Average length & Variance \\
\hline PSO & 46.77 & 39.55 & 42.67 & 3.11 \\
GWO & 45.98 & 38.57 & 39.88 & 4.02 \\
MGWO & 39.74 & 36.68 & 38.33 & 2.12 \\
\hline
\end{tabular}

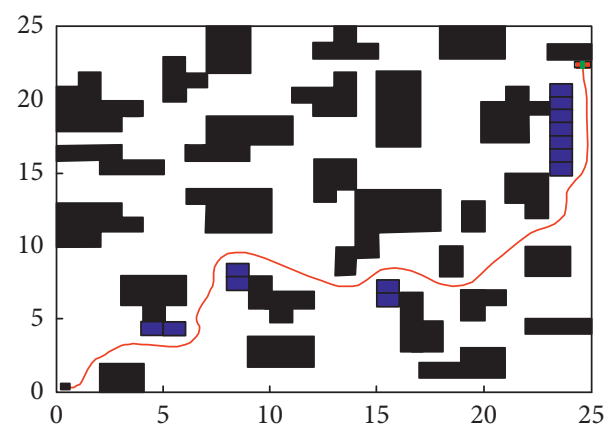

Figure 7: Path planned by the hybrid method after static obstacles added to the map.

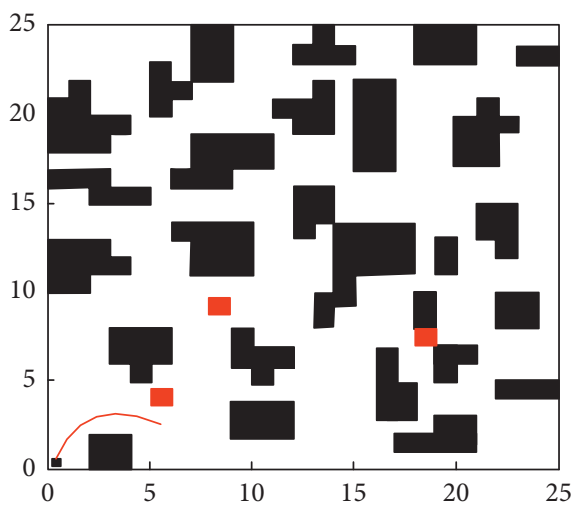

(a)

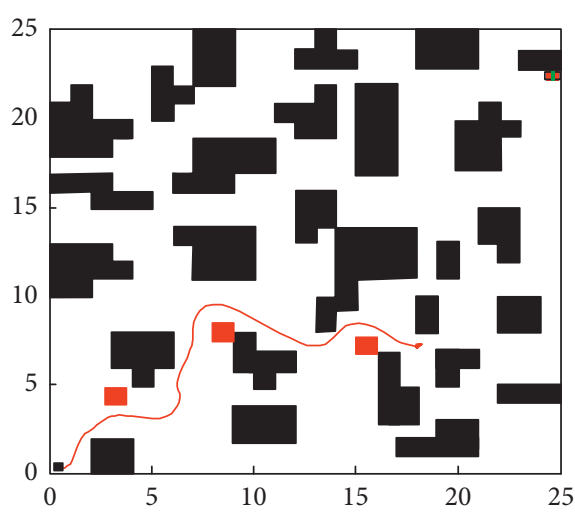

(c)

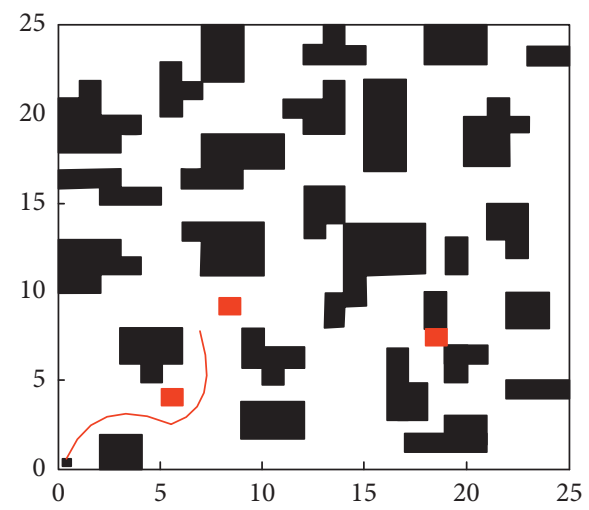

(b)

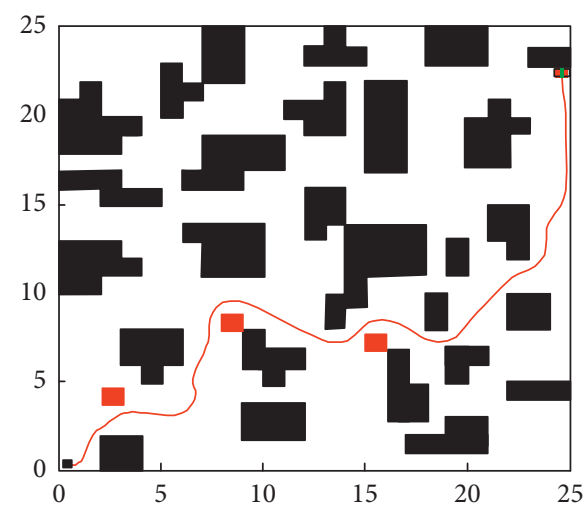

(d)

Figure 8: Path planned by the hybrid method after dynamic obstacles added to the map. (a) Hybrid path planning $a$. (b) Hybrid path planning b. (c) Hybrid path planning $c$. (d) Hybrid path planning $d$.

planning is carried out using the classic GWO, the proposed MGWO, and the particle swarm algorithm (PSO), respectively. In the simulation, for all the algorithms, the population size is set to 50, and the maximum number of iterations is set to 100 .
5.1.1. The Shortest Collision-Free Path. Figure 6 shows the path planning performance of the three algorithms in the grid environment. The quantified results of each of the three algorithms after 20 iterations are shown in Table 4 . It can be seen from the table that the proposed MGWO outperforms 
the other two algorithms in terms of the longest path, the shortest path, and the average path length.

Compared with PSO and classic GWO, the longest path planned by the proposed MGWO is $4.25 \mathrm{~m}$ and $4 \mathrm{~m}$ shorter, respectively, which is a reduction of $9.35 \%$ and $8.85 \%$, respectively. In terms of the shortest path length, the proposed MGWO has achieved a $3.08 \%$ and $1.58 \%$ reduction compared with PSO and classic GWO, respectively. Moreover, the average path length is also reduced by $7.13 \%$ and $2.88 \%$ by the proposed MGWO compared to PSO and classic GWO, respectively. In addition, the variance of the path length found by the proposed MGWO is more stable than the other algorithms.

5.2. Hybrid Path Planning Simulation. Static obstacles are added to the grid map to test the static obstacle avoidance capability of the proposed hybrid method; the 12 static obstacles added are shown in Figure 7 . In the figure, blue grids represent newly added obstacles; red lines indicate the moving path of the robot. Use the situation assessment method to plan the local path for the robot. After successfully avoiding the newly added obstacles, the robot continues to move along the global path until it reaches the endpoint.

Three dynamic obstacles are added to test the dynamic obstacle avoidance capability of the proposed hybrid method. The obstacles move horizontally, and the mobile robot successfully avoids the obstacles with the planned local path, indicating the path planning task is successfully accomplished. The planned path provided by the hybrid method is shown in Figure 8.

From the experimental results, it can be seen that compared with using only local path planning, the path planned by the proposed hybrid method is able to address the local minima problem and provide a path very close to the optimal path length. In addition, the simulation results also prove that the proposed hybrid method has dynamic obstacle avoidance capability; that is, the hybrid method can evade newly added static and dynamic obstacles and successfully reach the endpoint while guaranteeing an optimal path.

\section{Conclusion}

This article proposes a mobile robot path planning scheme based on a modified gray wolf optimization algorithm and situation assessment mechanism. Firstly, we propose the MGWO algorithm based on the GWO algorithm, which has the characteristics of good searching ability and robust performance. In MGWO, the population diversity is enhanced by logistic chaotic mapping, the balance between search and development capabilities of the GWO algorithm is achieved by the adaptive adjusting strategy of the control parameters, and a static weighting average strategy is used to update the population position and speed up convergence. Finally, combine the paths planned globally and locally to ensure that the situation assessment method can perform local path planning along the globally planned path.
Simulation results prove that the proposed scheme has higher optimization accuracy and stability than state-ofthe-art methods. The proposed MGWO algorithm can effectively reduce the path length and make the trajectory smoother. The hybrid method can ensure the optimal path to the greatest extent while taking into account the dynamic obstacle avoidance ability of the robot, which has more advantages than a single algorithm.

In the future, based on the scale of the tasks and battery capacity constraint of the robot, pathfinding can be performed based on task clustering, and large-scale tasks can be automatically and autonomously converted into time-by-step tasks so as to improve the applicability of path planning schemes in large-scale scenes.

\section{Data Availability}

The data used to support the findings of this study are included within the article.

\section{Conflicts of Interest}

The authors declare that there are no conflicts of interest regarding the publication of this article.

\section{References}

[1] F. Rubio, F. Valero, and C. Llopis-Albert, "A review of mobile robots: concepts, methods, theoretical framework, and applications," International Journal of Advanced Robotic Systems, vol. 16, no. 2, Article ID 1729881419839596, 2019.

[2] Z. Mohd Nayab and J. C. Mohanta, "Methodology for path planning and optimization of mobile robots: a review," Procedia Computer Science, vol. 133, pp. 141-152, 2018.

[3] J. Cheng, Hu Cheng, M. Q.-H. Meng, and H. Zhang, "Autonomous navigation by mobile robots in human environments: a survey," in Proceedings of the 2018 IEEE International Conference on Robotics and Biomimetics (ROBIO), December 2018.

[4] S. Campbell, N. O’Mahony, A. Carvalho, L. Krpalkova, D. Riordan, and J. Walsh, "Path planning techniques for mobile robots a review," in Proceedings of the 2020 6th International Conference on Mechatronics and Robotics Engineering (ICMRE), February 2020.

[5] B. Fu, L. Chen, Y. Zhou et al., "An improved $A^{*}$ algorithm for the industrial robot path planning with high success rate and short length," Robotics and Autonomous Systems, vol. 106, pp. 26-37, 2018.

[6] H. Ryu and Y. Park, "Improved informed $R R T^{*}$ using gridmap skeletonization for mobile robot path planning," International Journal of Precision Engineering and Manufacturing, vol. 20, no. 11, pp. 2033-2039, 2019.

[7] K. Akka and F. Khaber, "Mobile robot path planning using an improved ant colony optimization," International Journal of Advanced Robotic Systems, vol. 15, no. 3, Article ID $1729881418774673,2018$.

[8] E. Krell, A. Sheta, A. Balasubramanian, and S. King, "Collision-free autonomous robot navigation in unknown environments utilizing PSO for path planning," Journal of Artificial Intelligence and Soft Computing Research, vol. 9, 2019.

[9] J. Li, S. X. Yang, and Z. Xu, "A survey on robot path planning using bio-inspired algorithms," in Proceedings of the 2019 
IEEE International Conference on Robotics and Biomimetics (ROBIO), December 2019.

[10] S. Mirjalili, S. M. Mirjalili, and A. Lewis, "Grey wolf optimizer," Advances in Engineering Software, vol. 69, pp. 46-61, 2014.

[11] N. Toufan and A. Niknafs, "Robot path planning based on laser range finder and novel objective functions in grey wolf optimizer," SN Applied Sciences, vol. 2, no. 8, pp. 1-19, 2020.

[12] J. Oliveira, P. M. Oliveira, J. Boaventura-Cunha, and T. Pinho, "Chaos-based grey wolf optimizer for higher order sliding mode position control of a robotic manipulator," Nonlinear Dynamics, vol. 90, no. 2, pp. 1353-1362, 2017.

[13] K. Albina and S. G. Lee, "Hybrid stochastic exploration using grey wolf optimizer and coordinated multi-robot exploration algorithms," IEEE Access, vol. 7, pp. 14246-14255, 2019.

[14] U. Orozco-Rosas, O. Montiel, and R. Sepúlveda, "Mobile robot path planning using membrane evolutionary artificial potential field," Applied Soft Computing, vol. 77, pp. 236-251, 2019.

[15] Y. Zhang, Z. Liu, and L. Chang, "A new adaptive artificial potential field and rolling window method for mobile robot path planning," in Proceedings of the 2017 29th Chinese Control And Decision Conference (CCDC), May 2017.

[16] A. Stentz, The $D^{*}$ Algorithm for Real-Time Planning of Optimal Traverses, Carnegie-Mellon Univ Pittsburgh Pa Robotics Inst, Pittsburgh, Pennsylvania, 1994.

[17] M. M. Almasri, K. M. Elleithy, and A. M. Alajlan, "Development of efficient obstacle avoidance and line following mobile robot with the integration of fuzzy logic system in static and dynamic environments," in Proceedings of the 2016 IEEE Long Island Systems, Applications and Technology Conference (LISAT), April 2016.

[18] Z. Huang, C.-C. Shen, S. Doshi, N. Thomas, and H. Duong, "Fuzzy sets based team decision-making for cyber situation awareness," in Proceedings of the MILCOM 2016-2016 IEEE Military Communications Conference, November 2016.

[19] A. Arora, R. Fitch, and S. Sukkarieh, "An approach to autonomous science by modeling geological knowledge in a bayesian framework," in Proceedings of the 2017 IEEE/RSJ International Conference on Intelligent Robots and Systems (IROS), September 2017.

[20] Y. Zhou, Y. Tang, and X. Zhao, "A novel uncertainty management approach for air combat situation assessment based on improved belief entropy," Entropy, vol. 21, no. 5, p. 495, 2019.

[21] D. R. da Costa, O. Rene, T. Medrano, and E. Denis Leonel, "Route to chaos and some properties in the boundary crisis of a generalized logistic mapping," Physica A: Statistical Mechanics and its Applications, vol. 486, pp. 674-680, 2017. 